\title{
An Innovative Method for Low Cost, Autonomous Navigation for Low Earth Orbit Satellites
}

\author{
Julie Deutschmann \\ NASA Goddard Space Flight Center \\ Greenbelt, Maryland \\ julie.deutschmann@gsfc.nasa.gov \\ Rick Harman \\ NASA Goddard Space Flight Center \\ Greenbelt, Maryland \\ richard.harman@gsfc.nasa.gov \\ Itzhack Bar-Itzhack \\ Faculty of Aerospace Engineering \\ Technion-Israel Institute of Technology \\ Haifa, Israel
}

\begin{abstract}
An innovative approach to autonomous attitude, trajectory, and rate estimation is presented for low earth orbit (LEO) satellites which relies on magnetometers and sun sensors. These two sensors are reliable, inexpensive, and are used routinely in LEO missions for attitude determination and control. An extended Kalman filter (EKF) is developed from two existing systems, one which uses an EKF to estimate attitude and trajectory using magnetometer and gyro data and a second pseudo-linear filter which estimates rotation rate using magnetometer and sun sensor data. The theoretical background of the combined system is presented along with test results from noisy, simulated sensor data.
\end{abstract}

\section{INTRODUCTION}

Most missions supported by the NASA Goddard Space Flight Center (NASA/GSFC) have an attitude and orbit determination requirement. In most cases, the attitude estimation is performed onboard the satellite. However, the orbit determination is performed primarily on the ground post pass. Efforts are underway to provide for spacecraft onboard autonomous orbit determination. However, these efforts can be somewhat expensive and have limited availability which makes them less attractive to the multitude of missions being launched with very modest attitude and orbit requirements as well as modest budget.

Previous research demonstrated an approach to attitude and trajectory estimation using only magnetic field data and rate data ${ }^{3}$. The estimation is performed simultaneously using an EKF, a well known algorithm used extensively in onboard applications. The magnetic field is measured on a satellite by a magnetometer, an inexpensive and reliable sensor flown on virtually all LEO satellites. The system has been developed and successfully tested in a postprocessing mode using magnetometer and gyro data from four satellites supported by the Guidance, Navigation, and Control Center at the NASA/GSFC. However, the rate data required by this algorithm is provided by a gyro, which can be costly.

\footnotetext{
- Sophie and William Shamban Professor of Aerospace Engineering, AIAA Associate Fellow, LEEE Fellow 'Copyright $\odot 1998$ by the American Institute of Aeronautics and Astronautics, Inc. No copyright is asserted in the United States under Title 17, U.S. Code. The U.S. Government has a royalty-free license to exercise all rights under the copyright claimed herein for Governmental Purposes. All other rights are reserved by the copyright owner.
} 
In order for this system to be truly low cost, an alternative source for rate data must be utilized. An independent system which estimates spacecraft rate has been successfully developed and tested using magnetometer data and sun sensor data, along with the known attitude ${ }^{2}$. This system is much less costly than a gyro since it is software based and uses sensors which are traditionally flown on low earth orbiting missions. Both systems have been combined into one low cost attitude, rate, and orbit estimation system and preliminary test results have been conducted ${ }^{3}$.

The resulting system is expected to provide low cost navigation, i.e. attitude, orbit, and rates, for low earth orbit satellites. The system relies on existing hardware, namely, magnetometers and sun sensors. Magnetometers are carried on virtually all low earth orbit satellites. There has been only 1 reported failure of a magnetometer for missions supported by NASAVGSFC. Sun sensors are also extremely reliable. Both sensors are currently available and most importantly, they are flight qualifin Comparable systems, such as the Global fosition/Wystem, are considerably more expensive and flight qualified receivers which can perform attitude determination are not readily available. Any LEO mission, whether commercial or government, with coarse accuracy requirements or desiring an inexpensive backup method for attitude, rate, and orbit estimation can use this system, provided the satellite has an onboard computer. The impact on the onboard processing should not be significantly more than current onboard processing and can be accomplished with current computing technology. Furthermore, utilizing onboard processing reduces the cost of ground operations.

In this work, the EKF developed to estimate the orbit, attitude, and rates and initially tested in Reference 3 is presented. The algorithm is summarized and the results of further testing are included. The testing consisted of noisy, simulated data spans up to 48 hours in length; results with an attitude maneuver are included as well.

\section{THEORETICAL BACKGROUND}

The assumed models of the EKF are given as:

System model:

$$
\underline{X}_{k}(t)=\underline{f}\left(\underline{X}_{k}(t), t_{k}\right)+\underline{u}_{k}(t)
$$

Measurement model

$$
\underline{z}_{k}=\underline{h_{k}}\left(\underline{X}\left(t_{k}\right)\right)+\underline{\eta}_{k}
$$

\section{Update Stage}

The linearization of equation (2) results in

$$
\underline{z}_{k}=H_{k} \underline{x}_{k}+\underline{\eta}_{k}
$$

where $\mathrm{H}_{k}$ is the measurement matrix of the combined filter at time $\mathfrak{t}_{\mathbf{k}}$. $\mathrm{H}_{\mathbf{k}}$ is composed of sub-matrices which reflect the dependence of the effective measurement $\underline{z}_{k}$ on the state vector, $\underline{x}_{\mathbf{k}}$ which contains the orbital elements, the angular attitude error, and the angular velocity.

$$
\underline{\mathbf{x}}_{\mathbf{x}}^{\mathrm{T}}=\left[\mathbf{a}, \mathbf{e}, \mathbf{i}, \Omega, \mathbf{w}, \theta, \mathbf{C}_{d}, \underline{\phi}, \underline{\omega}\right]
$$

where:

$$
\begin{aligned}
& a=\text { semi-major axis } \\
& e=\text { eccentricity } \\
& i=\text { inclination } \\
& \Omega=\text { right ascension of ascending node } \\
& w=\text { argument of perigee } \\
& \theta=\text { true anomaly } \\
& C_{d}=\text { drag coefficient } \\
& \Phi=\text { attitude error } \\
& \underline{\omega}=\text { rotation rate }
\end{aligned}
$$

The measurement matrix is

$$
H_{k}=\left[\begin{array}{ccc}
H_{0, k} & H_{a, k} & 0 \\
0 & 0 & {\left[\underline{b}_{k} \times\right]}
\end{array}\right]
$$

Where $\mathrm{H}_{0, k}$ and $\mathrm{H}_{\mathrm{a}, \mathrm{k}}$ are, respectively, the submatrices reflecting the dependence of the orbital components ${ }^{4}$ and the attitude ${ }^{5}$ on the effective measurement. The matrix $\left[b_{k} x\right]$ is a skew symmetric matrix composed of the elements of the measured vector, $\underline{b}$. The development of the dependence of the angular velocity on the effective measurement follows.

The effective measurement, $\underline{z}_{k}$ contains two elements, $\underline{z}_{1, k}$ and $\underline{z}_{2, k}$. The first is the difference between the measured vector and computed reference vector ${ }^{1}$ and the second element is the difference in the derivatives of the measured vector and computed reference vector ${ }^{2}$. Taking the derivatives of the computed and measured vectors brings in a dependence on the angular velocity through the formula:

$$
D_{v, k}^{I} \underline{\underline{I}}_{k}=\underline{\dot{b}}_{k}+\underline{\omega}_{k} \times \underline{\mathbf{b}}_{k}
$$


where: $\underline{1}_{\mathrm{k}}=$ angular velocity vector

$$
\begin{aligned}
\underline{r}_{k}= & \text { computed reference vector resolved in } \\
& \text { inertial coordinates } \\
D_{v . k}^{l}= & \text { transformation matrix that transforms } \\
& \text { vectors from inertial to vehicle (body) } \\
& \text { coordinates } \\
\underline{b}_{k}= & \text { observed vector resolved in body } \\
& \text { coordinates }
\end{aligned}
$$

Note that $\underline{r}_{k}$ and $\underline{b}_{x}$ are the components of the same abstract vector. Incorporating the noise into the reference and observed vectors, (6) can be written as

$\underline{\underline{b}}_{k}-D_{v, k}^{I} \dot{\underline{r}}_{k}=\left[\underline{b}_{k} \times \underline{\omega}_{k}+\left[\underline{\eta}_{b, k} \times \underline{\omega}_{k}-\underline{\eta}_{\dot{b}, k}+D_{v, k}^{I} \underline{\eta}_{i, k}\right.\right.$

where: $\underline{p}_{b, k}=$ measurement vector noise at time $t_{k}$

$$
\begin{aligned}
\underline{\eta}_{\dot{b}, k}= & \left(\underline{\eta}_{b, k}-\eta_{b, k-1}\right) / \Delta \\
\underline{\eta}_{i, k}= & \text { reference vector derivative noise } \\
{\left[\underline{b}_{k} x\right]=} & \text { skew symmetric matrix composed of the } \\
& \text { elements of } \underline{b}_{k}, \text { which is entered into } H_{k} \\
& \text { above } \\
\Delta= & \text { time between measurements }
\end{aligned}
$$

In view of (6), the second element of the effective measurement is then formally defined by the following relationship

$$
\underline{z}_{2, k} \doteq\left(\underline{b}_{k}-D_{v, k}^{1} \dot{\underline{r}}_{k}\right)-\left[\underline{b}_{k} \times \underline{\omega}_{\text {est }, k}\right.
$$

where: $\underline{\omega}_{\text {ess }, x}=$ current estimated rate

Assuming the noise from the reference vector to be zero, the noise terms in (7) can be combined into

$$
\underline{\eta}_{d, k}=\left[-\underline{\omega}_{k} \times \underline{\eta}_{b, k}-\underline{\eta}_{\dot{b}, k}\right.
$$

The measurement noise, $\underline{\eta}_{b, k}$, associated with $\underline{z}_{1, k}$ is augmented with the noise $\eta_{d, k}$ into the noise vector, $\eta_{k}$, of (3). In order to use this in the filter the covariance matrix $R_{k}$ is computed as

$$
\begin{aligned}
& \mathrm{R}_{\mathrm{k}}=\mathrm{E}\left\{\left[\begin{array}{l}
\underline{\underline{\eta}}_{\mathrm{b}, \mathrm{k}} \\
\underline{\eta}_{d, k}
\end{array}\right]\left[\begin{array}{lll}
\underline{\eta}_{b, k} & \underline{\eta}_{d, k} & \mathrm{~T}
\end{array}\right]\right\}- \\
& \mathrm{E}\left\{\left[\begin{array}{l}
\underline{\underline{\eta}}_{\mathrm{b}, \mathrm{k}} \\
\underline{\underline{\eta}}_{\mathrm{d}, \mathrm{k}}
\end{array}\right]\right\} \mathrm{E}\left\{\left[\begin{array}{lll}
\underline{\eta}_{\mathrm{b}, \mathrm{k}} & \underline{\underline{\eta}}_{\mathrm{d}, \mathrm{k}}
\end{array}\right]\right\}
\end{aligned}
$$

If the sensor is calibrated such that the measurements have no bias $E\left\{\eta_{b, k}\right\}$ and $E\left\{\eta_{a k k}\right\}$ are zero and $R_{k}$ becomes

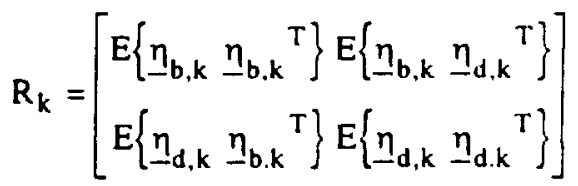

The matrix $E\left\{\underline{n}_{b, k} \underline{\eta}_{b, k}{ }^{\top}\right\}$ is the noise covariance matrix for the measurement, i.e. $R_{\text {TAM }}$ for the magnetometer. Based on the assumption that the $\eta_{k, k}$ and $\eta_{b, k-1}$ are uncorrelated, $E\left\{\eta_{d, k} \eta_{d, k}{ }^{\top}\right\}$ becomes

$$
E\left\{\underline{\eta}_{d, k} \underline{\eta}_{d, k}{ }^{T}\right\}=D_{k} R_{\text {TAM }} D_{k}{ }^{T}+\left(1 / \Delta^{2}\right) R_{T A M}
$$

where the matrix $D_{k}$ is computed as

$$
D_{k}=\left[\underline{\omega}_{\text {est }, k} \times\right]+(1 / \Delta) I
$$

and $I$ is a $3 \times 3$ identity matrix. The noise covariance matrix then becomes

$$
R_{k}=\left[\begin{array}{lc}
R_{T A M} & -R_{T A M} D_{k}{ }^{T} \\
-D_{k} R_{T A M} & D_{k} R_{T A M} D_{k}{ }^{T}+\left(1 / \Delta^{2}\right) R_{T A M}
\end{array}\right]
$$

The update of the state vector and covariance matrix is performed using the following equations

$$
\begin{gathered}
\underline{x}_{s}(+)=\underline{x}_{k}(-)+K_{k} \underline{Z}_{k} \\
P_{k}(+)=\left(I-K_{k} H_{k}\right) P_{k}(-)\left(I-K_{k} H_{k}\right)^{T}+K_{k} R_{k} K_{k}{ }^{T}
\end{gathered}
$$

where the gain matrix, $K_{k}$ is computed as

$$
\mathrm{K}_{k}=\mathrm{P}_{k}(-) \mathrm{H}_{\mathbf{k}}{ }^{\mathrm{T}}\left[\mathrm{H}_{\mathbf{k}} \mathrm{P}_{\mathrm{k}}(-) \mathrm{H}_{\mathrm{k}}+\mathrm{R}_{\mathrm{k}}\right]^{-1}
$$

The state vector, $\underline{x}_{k}$, is the internal state used by the EKF. This form is used internally to estimate the angular error in the attitude (in addition to the other state vector elements) which is then converted to a normalized quaternion for propagation. This is the socalled 'multiplicative' approach'.

The above derivation is valid for a magnetometer. For another sensor, such as a sun sensor, the following changes must be made. First, since another sensor is not influenced by the orbit, the measurement matrix in (5) is replaced with

$$
H_{k}=\left[\begin{array}{lll}
0 & H_{a, k} & 0 \\
0 & 0 & {\left[\underline{b}_{k} \times\right]}
\end{array}\right]
$$


where $\underline{b}_{k}$ is the measured vector. The effective measurement, $\underline{z}_{k}$. is based on the sensor measurements of the given sensor and is computed as for the magnetometer. The computation of $R_{k}$ is as given in (14) with $R_{\text {TAM }}$ replaced by the noise covariance matrix of the given sensor. Based on the results of Reference 2 and 5 , the $3^{\text {rd }}$ and $6^{\text {th }}$ rows of $H_{k}$ in (17) above and the corresponding rows and columns of $R_{k}$ are removed. This is to prevent singularities.

\section{Propagation stage}

The propagation of the state estimate, based on equation 1 is performed as

$$
\underline{\hat{\mathbf{X}}}_{k}=\mathrm{f}\left(\underline{\hat{X}}_{k}(t), t_{k}\right)
$$

Where $\underline{\hat{X}}_{k}$ is the state vector described above except that it contains the estimated quaternion computed from the angular error estimate. The updated estimate of the state vector is propagated from time $t_{k}$ to $t_{k+1}$ as a solution of the dynamics equation. The orbital dynamics equation is non-linear and describes a central force including both $\mathrm{J} 2$ effects and $\mathrm{drag}^{3}$. The orbital elements are propagated using a $4^{\text {th }}$ order Runge-Kutta integration. The differential equation which governs the propagation of the quaternion is linear and is dependent on the estimation of the spacecraft rotation rate $^{6}$. The spacecraft dynamics equation is also nonlinear and the rate estimate is propagated using Euler's equation. The external torques, momentum generated by the momentum wheels, and inertia tensor of the spacecraft are required. The numerical solution is obtained with the MATLAB ODE function.

The propagation of the covariance matrix is performed using the following equation

$$
P_{k+1}(-)=A_{k}\left(\underline{\hat{X}}_{k}(+)\right) P_{k}(+) A_{k}^{T}\left(\underline{\hat{X}}_{k}(+)\right)+Q_{k}
$$

where $Q_{k}$ is the covariance matrix of $\underline{v}_{k}$ of (1) and $A_{k}$ is the approximated transition matrix. $A_{k}$ is computed using the following first order Taylor series expansion

$$
A_{k}=I+F_{k} \Delta T
$$

where $\Delta \mathrm{T}$ is the propagation time step. The Jacobian $F_{k}$, computed from $f\left(X_{k}(t), t_{k}\right)$, is derived for the orbital dynamics in Reference 4, for the attitude dynamics in Reference 5, and for the spacecraft dynamics in Reference 2 .

\section{RESULTS}

The algorithm is tested with noisy, simulated magnetometer and sun sensor data. The simulated data is based on the Rossi X-ray Timing Explorer (RXTE) satellite which is in a near circular $560 \mathrm{~km}$ altitude, 23 degrees inclination orbit. The length of the data span is $\mathbf{4 8}$ hours. Table 1 lists the 'true' state variables and the initial state used in the EKF.

Table 1. True and Initial State Parameters

\begin{tabular}{ccc} 
Parameter & $\underline{\text { Truth }}$ & $\underline{\text { Initial }}$ \\
\hline $\mathrm{a}(\mathrm{km})$ & 6956.7 & 7956.7 \\
$\mathrm{e}$ & 0.00197 & 0.002 \\
$\mathrm{i}(\mathrm{deg})$ & 22.96 & 22.5 \\
$\Omega(\mathrm{deg})$ & 109.74 & 110.74 \\
$\mathrm{w}(\mathrm{deg})$ & 220.04 & 225.036 \\
$\mathrm{q}(\mathrm{deg})$ & 18.19 & 28.19 \\
$\mathrm{C}_{\mathrm{d}}$ & 2.2 & 0 \\
$\mathrm{q}(1)$ & 0 & 0.0454 \\
$\mathrm{q}(2)$ & 0 & 0.0416 \\
$\mathrm{q}(3$ & 0 & 0.0454 \\
$\mathrm{q}(4)$ & 1 & 0.9971 \\
$\omega_{\mathrm{x}}$ & 0 & 0.1 \\
$(\mathrm{deg} / \mathrm{sec})$ & & 0.1 \\
$\omega_{\mathrm{y}}$ & 0 & 0.1 \\
$(\mathrm{deg} / \mathrm{sec})$ & & \\
$\omega_{\mathrm{z}}$ & 0 & \\
$(\mathrm{deg} / \mathrm{sec})$ & &
\end{tabular}

Results of three tests are presented. The first test (TEST 1) contains inertial data with continuous coverage of both magnetometer and sun sensor data. In the second test (TEST 2) an attitude maneuver is inserted after 5 hours. The maneuver lasts 10 minutes with a rate of $0.15 \mathrm{deg} / \mathrm{sec}$ applied to the $z$ axis. No control data is input to the EKF during the maneuver. In the final test (TEST 3 ) the sun sensor is occulted for 60 percent of each orbit.

Figure 1 shows the RSS position error from the first test. The final average errors are approximately 20-30 $\mathrm{km}$. It appears that the estimate is still converging. The final velocity errors are less than $0.025 \mathrm{~km} / \mathrm{sec}$. Figure 2 shows the attitude estimation errors and Figure 3 shows the rate estimates. The attitude errors are less than 2 degrees per axis. The rate errors are centered around zero and converge to less than 0.003 $\mathrm{deg} / \mathrm{sec}$ on each axis.

Figures 4 through 6 show the RSS position, attitude errors, and rate estimates, respectively, for the second 
test which included the attitude mancuver at 5 hours or approximately 3.1 orbits. The position estimates improve with the final average RSS position errors approximately $20 \mathrm{~km}$. The velocity estimates exhibit similar behavior to the position estimates with final errors comparable to the first test. Since the maneuver was 90 degrees the attitude errors are comparable to the first test with a change of axes. The attitude errors average to approximately zero. The rate estimate is comparable as well. Figure 7 shows an expanded view of the $z$ axis rate at the time of the maneuver. The estimate follows the maneuver.

The results of the final test are presented in Figures 8 through 10 . The attitude maneuver again occurs at 5 hours. The RSS position error, shown in Figure 7, is comparable to Figure 4 with slightly greater oscillations, but a slightly lower final average. The same is true, again, for the velocity errors. The noise covariance associated with the magnetometer was decreased for this test, resulting in the improved position estimates. The attitude errors degrade at times with the loss of the sun data as do the rate estimates. But overall, the filter results are reasonable considering the estimates are based on magnetometer data alone for 60 percent of each orbit.

\section{CONCLUSIONS}

Results of three tests of the EKF are presented. The first consisted of 48 hours of noisy, simulated magnetometer and sun sensor data with an inertial attitude. An attitude maneuver was inserted for the second test, and finally, in the third test the sun sensor was occulted for 60 percent of the orbit. In all cases the EKF simultaneously estimated orbit, attitude, and rates. Starting from initial errors of over $1000 \mathrm{~km}$ and $2 \mathrm{~km} / \mathrm{sec}$ in position and velocity, 5 degrees per axis in attitude, and $0.1 \mathrm{deg} / \mathrm{sec}$ per axis for the rate, the final average RSS position and velocity errors are approximately $20 \mathrm{~km}$ and $0.02 \mathrm{~km} / \mathrm{sec}$, respectively. The attitude errors are less than 2 degrees RSS, and the rates average less than $0.003 \mathrm{deg} / \mathrm{sec}$ per axis. The EKF was able to detect and follow the attitude maneuver, without any control data. Finally, the EKF was able to perform without continuous sun sensor data.

Future tests with longer data spans, real spacecraft data. and inflight test data are planned. In addition, further tests will be conducted to determine the minimum sun coverage needed per orbit in order to achieve reasonable estimates.

\section{REFERENCES}

1. Deutschmann, J. and Bar-Itzhack, I., "Attitude and Trajectory Estimation Using Earth Magnetic Field Data", Paper No. AIAA-96-3631, presented at AIAAAAS Astrodynamics Conference, San Diego, CA, July 29-31, 1996.

2. R.R. Harman and I.Y. Bar-Itzhack, "The Use of Pseudo-Linear and SDARE Filtering for Satellite Angular-Rate Estimation", AIAA Guidance, Navigation, and Control Conference, Boston, MA, August 10-12, 1998.

3. Deutschmann, J., R. Harman, I. Bar-Itzhack, "A Low Cost Approach to Simultaneous Orbit, Attitude, and Rate Estimation Using an Extended Kalman Filter", $13^{\text {th }}$ International Symposium on Space Flight Dynamics, NASA Goddard Space Flight Center, May 11-15, 1998.

4. Shorshi, G., and Bar-Itzhack, I., "Satellite Autonomous Navigation Based on Magnetic Field Measurements", TAE No. 714, Technion-Israel Institute of Tech., Haifa, Israel, April 1994.

5. Deutschmann, J., Bar-Itzhack, I., and Rokni, M., "Comparison and Testing of Extended Kalman Filters for Attitude Estimation of the Earth Radiation Budget Satellite", presented at the GSFC FDD Flight Mechanics/Estimation Theory Symposium 1990, NASA GSFC, Greenbelt , Maryland, May 22-24, 1990.

6. Wertz, James, R., Spacecraft Attitude Determination and Control, D. Reidel Publishing Company, Dordrecht, Holland, 1984. 


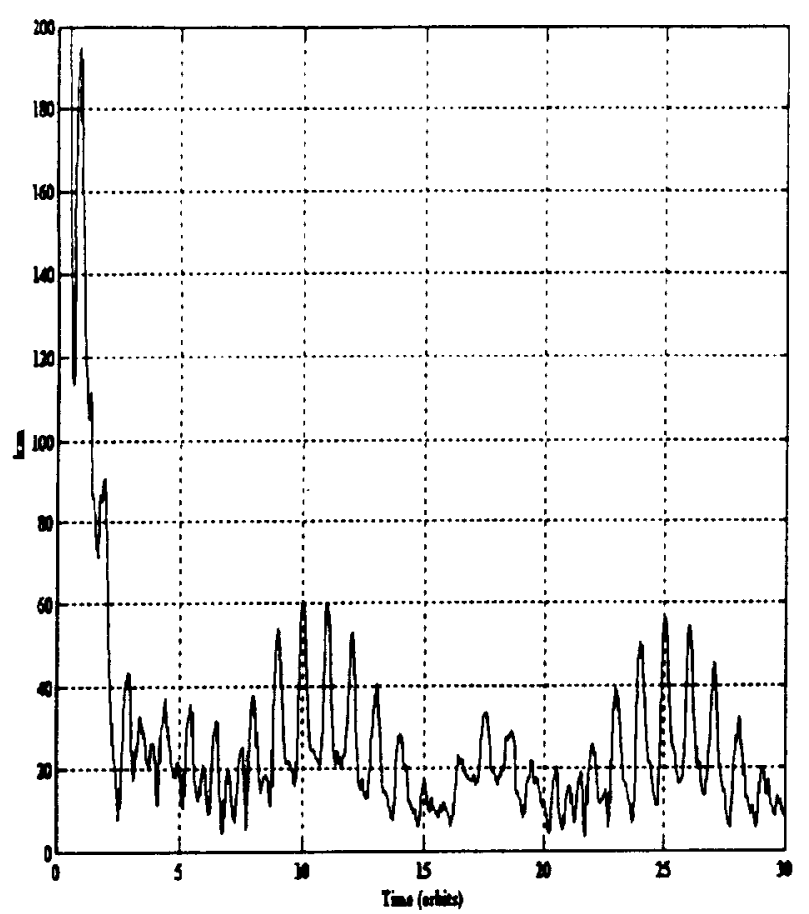

Fiqure 1. RSS Position Error - TEST 1
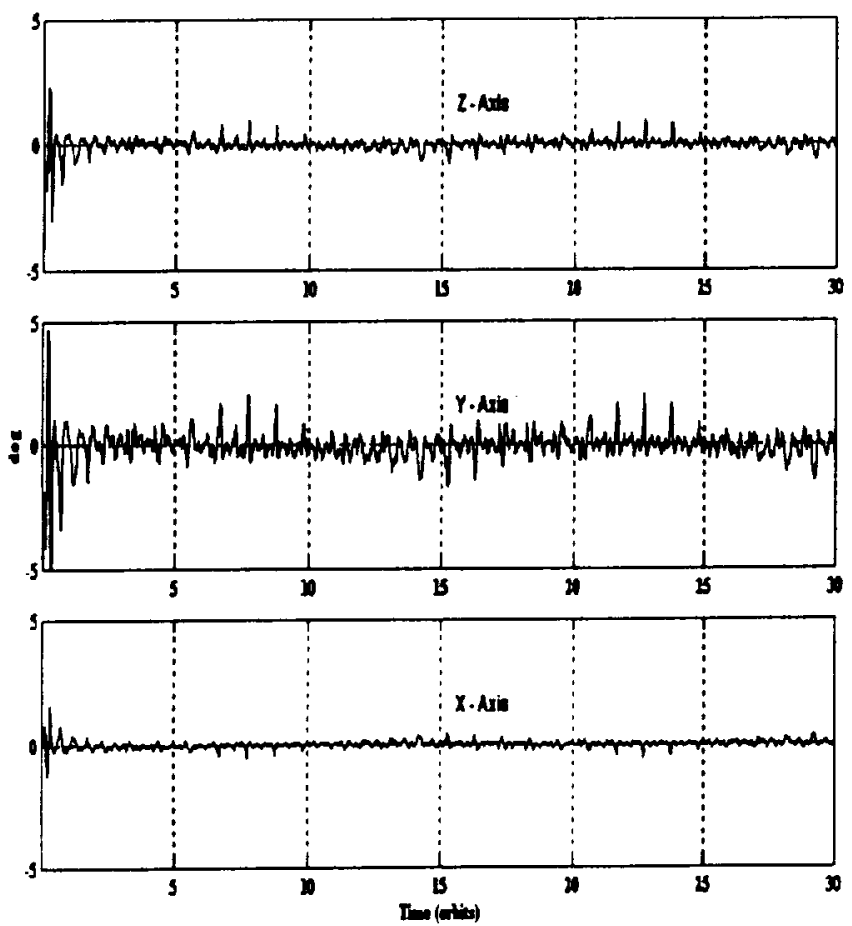

Fiqure 2. Attitude Errors - TEST 1
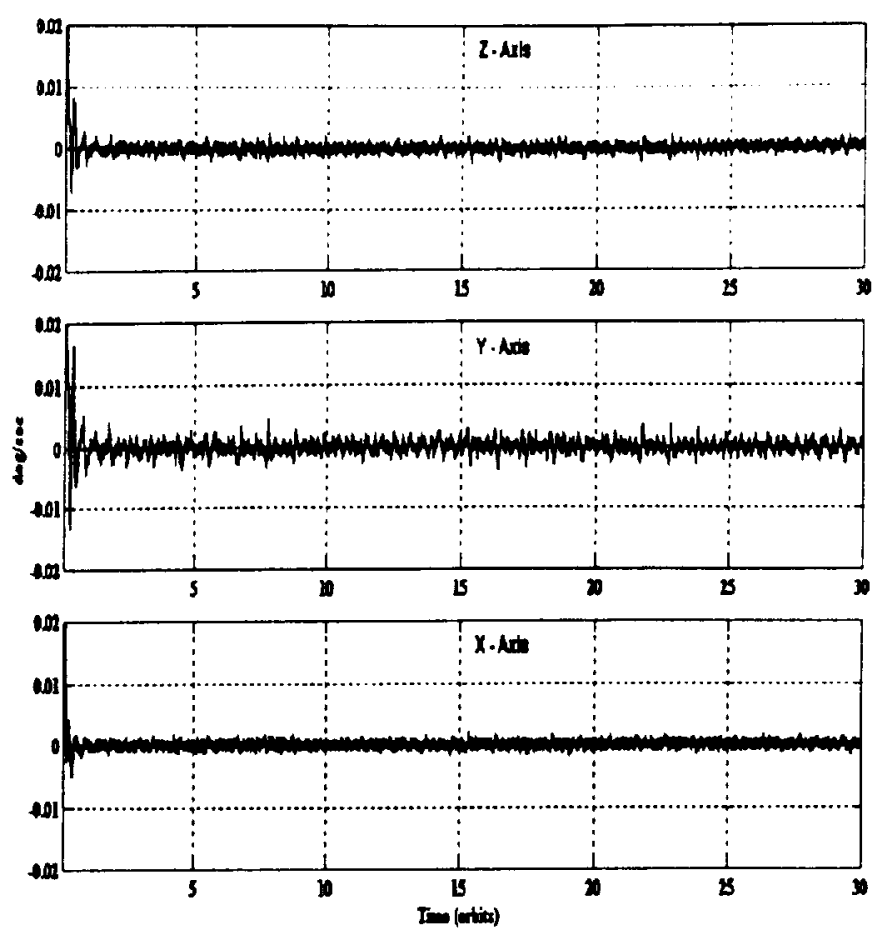

Fipure 3. Rate Estimates - TEST 1

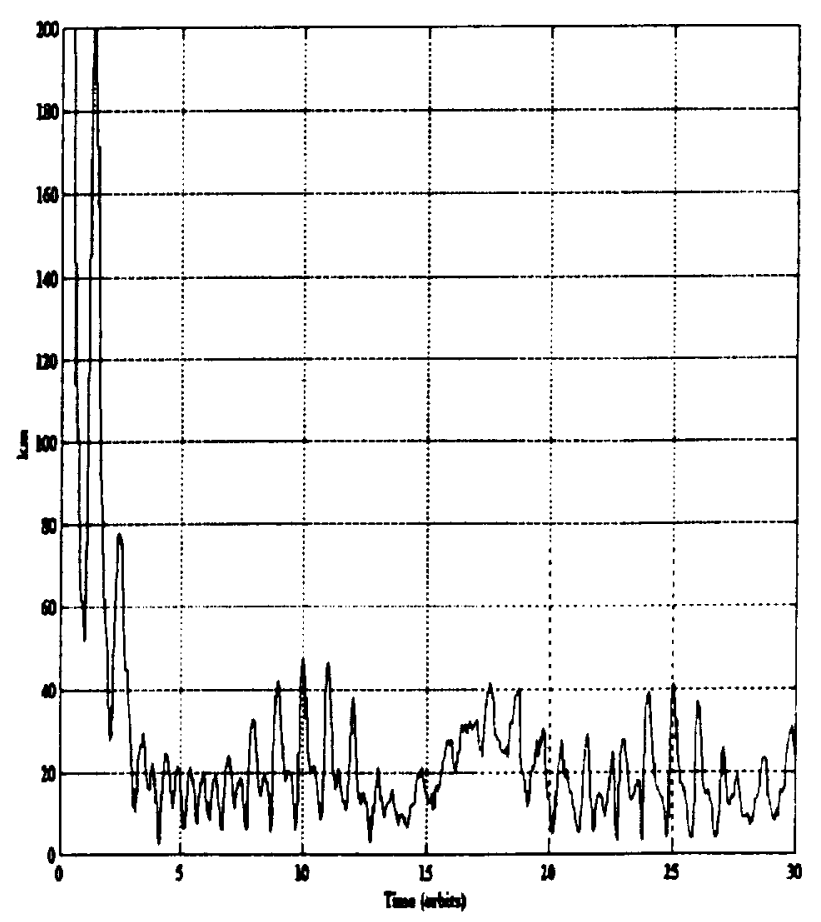

Fipure 4. RSS Position Error - TEST 2 

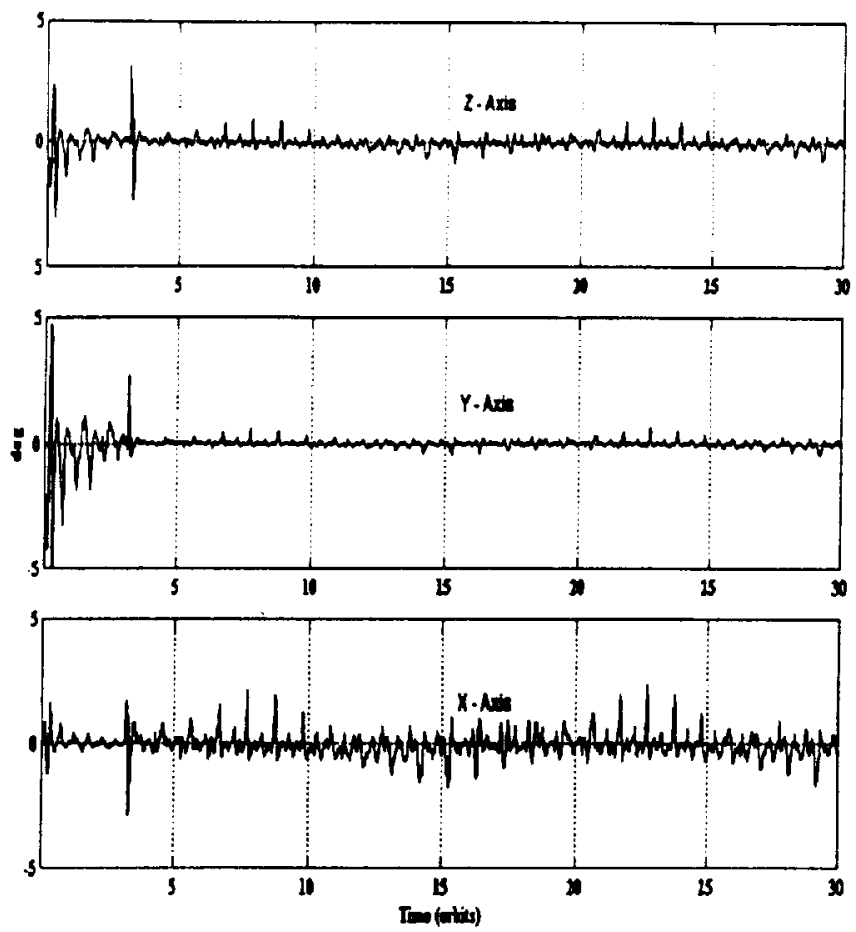

Fipure 5. Attitude Errors - TEST 2

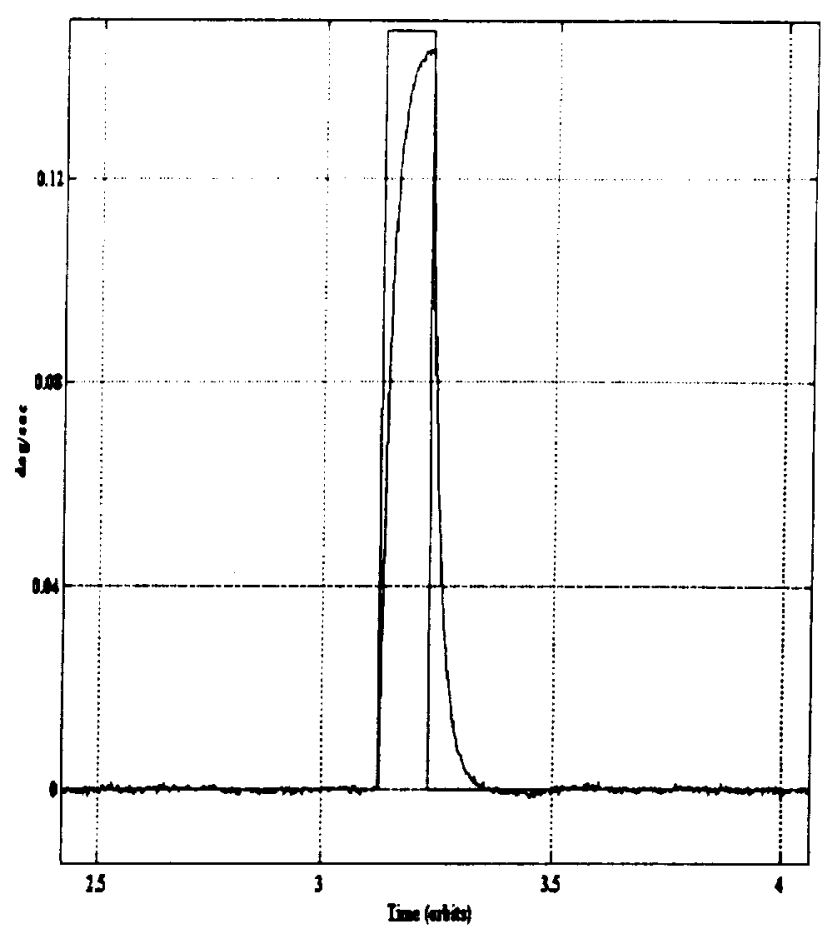

Figure 7. Z Axis Rate Estimate and True Rate - TEST 3

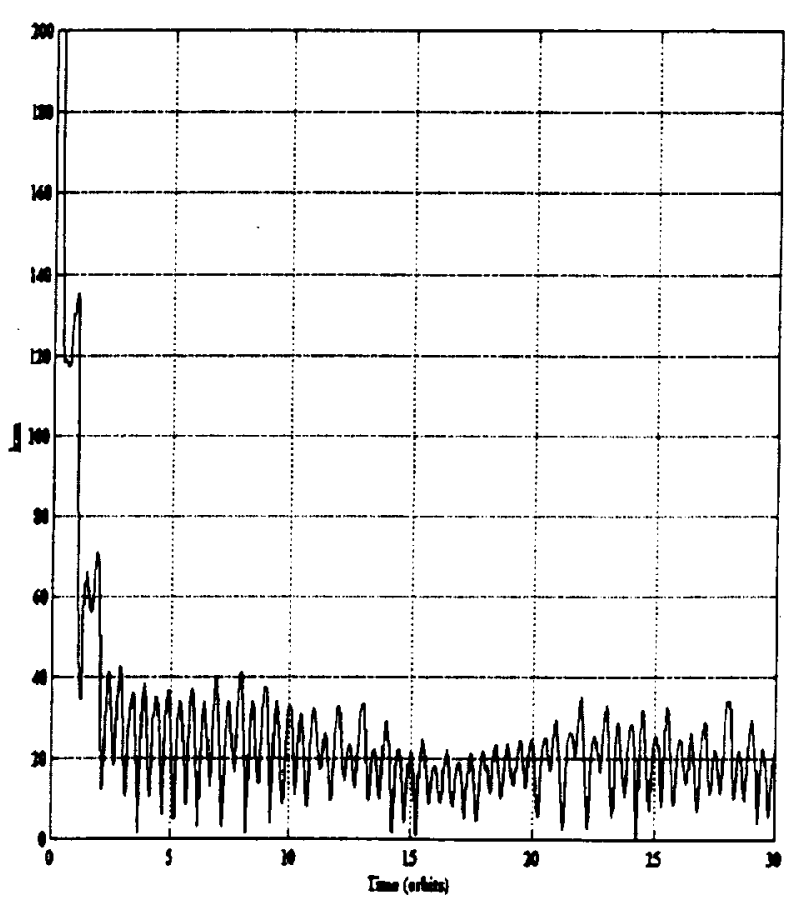

Fiqure 8. RSS Position Error - TEST 3 

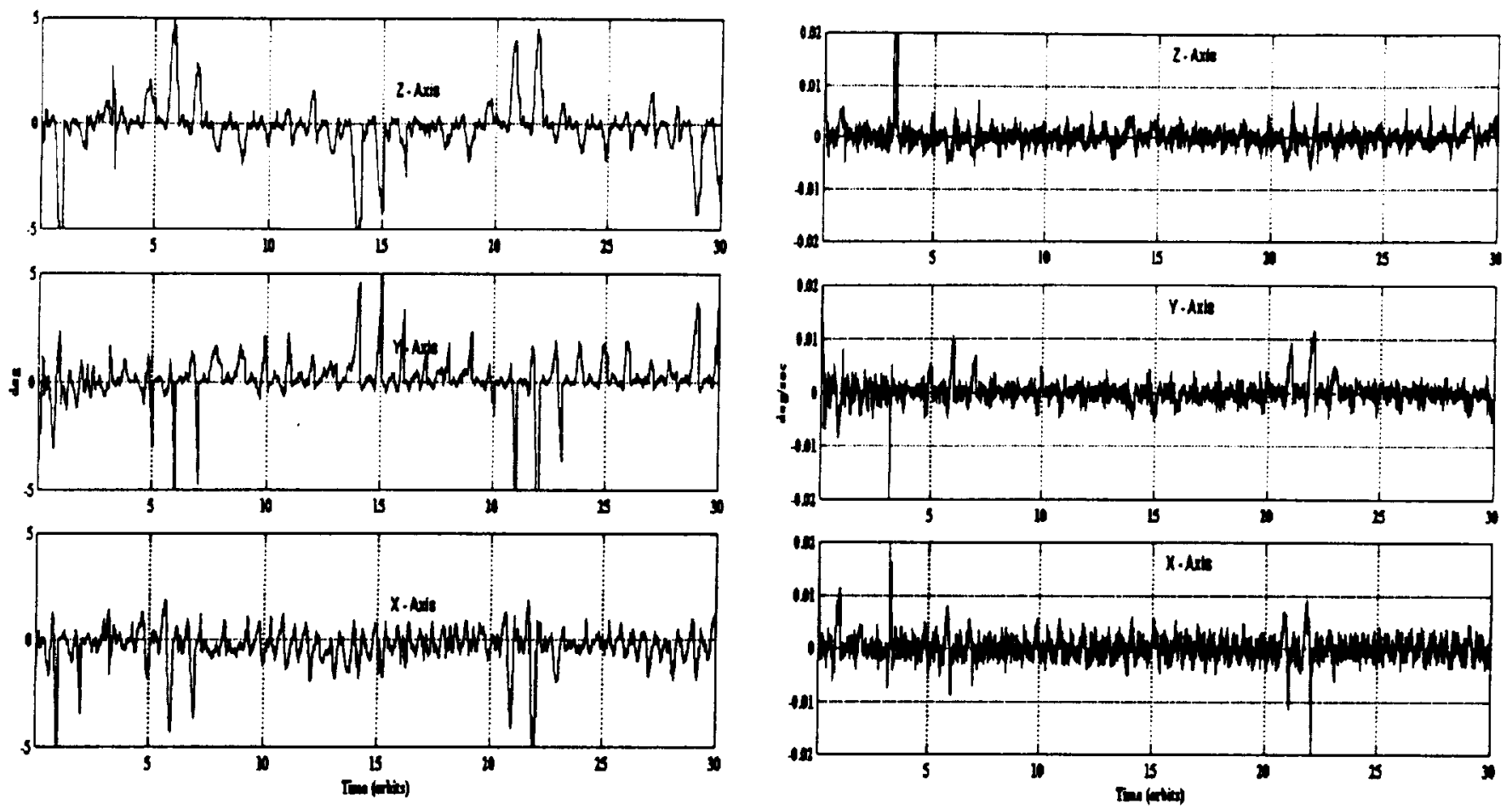

Fiqure 4 Attitude Errors - TEST 3

Figure 10. Rate Estimates - TEST 3. 